\title{
Diálogo de saberes con redes sociales caso específico atractivo turístico los currucuyes
}

\section{Dialogue of knowledge with social networks specific case of the currucuyes tourist attraction}

\author{
DOI: $10.46932 / s f j d v 3 n 1-110$
}

Received in: Jan 30st, 2021

Accepted in: Feb 1th, 2022

\author{
Eugenia Grosso Molano MS \\ Profesor de la Escuela de Administración Turística y Hotelera en la Universidad Pedagógica y \\ Tecnológica de Colombia, Facultad Seccional Duitama Boyacá. \\ E-mail: eugenia.grosso@uptc.edu.co \\ Margie Julieth Siabato Castillo \\ Estudiante de Administración Turística y Hotelera en la Universidad Pedagógica y Tecnológica de \\ Colombia, Facultad Seccional Duitama Boyacá. \\ E-mail: margie.siabato@uptc.edu.co
}

\begin{abstract}
RESUMEN
Hoy en día el turismo ha tomado gran importancia en el departamento de Boyacá Colombia, debido a sus grandes riquezas naturales y su diversidad cultural con relación mitos, leyendas y tradiciones, a través del diálogo de saberes se comprende la problemática y necesidades que tiene la población mediante la reflexión y discusión entre los diferentes actores involucrados, dan a conocer experiencias a través de la utilización de las TIC, específicamente por medio de las redes sociales, establecen nuevas formas de interacción y relacionarse de forma eficaz, oportuna y actualizada acerca del lugar, el turista planea sus viajes y diseña sus propios itinerarios, comparte sus vivencias y experiencias; la investigación se realiza con un enfoque cualitativo empleando como instrumentos la observación directa, las encuestas, obteniéndose como resultado que muchos turistas encontraron interesante el mirador Ecoturístico los Currucuyes, se tienen varios seguidores e igualmente han visitado el lugar, realizaron actividades complementarias en los municipios aledaños y se aplicó la gestión del conocimiento a través del diálogo de saberes entre el conocimiento tácito al explícito.
\end{abstract}

Palabras clave: turismo sostenible, diálogo de saberes, TIC, redes sociales, ecoturismo.

\begin{abstract}
Today tourism has taken great importance in the department of Boyacá Colombia, due to its great natural wealth and its cultural diversity in relation to myths, legends and traditions, through the dialogue of knowledge the problems and needs of the population are understood through reflection and discussion among the different actors involved, they share experiences through the use of ICT, specifically through social networks, establish new forms of interaction and relate effectively, timely and updated about the place, tourists plan their trips and design their own itineraries, share their experiences and experiences; The research is carried out with a qualitative approach using direct observation and surveys as instruments, obtaining as a result that many tourists found the Ecotourism viewpoint Los Currucuyes interesting, there are several followers and they have also visited the place, they carried out complementary activities in the surrounding municipalities and knowledge management was applied through the dialogue of knowledge between tacit and explicit knowledge.
\end{abstract}


Keywords: sustainable tourism, dialogue of knowledge, ICT, social networks, ecotourism.

\section{INTRODUCCIÓN}

La gran diversidad de climas que tiene el departamento y atractivos con inigualable belleza como es el Mirador ecoturístico los Currucuyes, encuentran una gran variedad de bosques nativos para ser visitados por grupos de amigos, familiares. El turismo junto a las Tecnologías de la Información y la Comunicación (TIC), han venido cambiando e impactando la forma de acceder y promover la industria del turismo cada vez más se hace necesario diseñar contenidos digitales queden a conocer destinos turísticos que evidencie los grandes paisajes naturales con que cuenta nuestro departamento.

Cada vez más está cobrando importancia el proceso por la conservación del territorio y sus saberes ancestrales que cambian constantemente ya que cada generación va imprimiendo su conocimiento, en donde se hace necesario incorporar el dialogo de saberes para conocer las problemáticas y trabajar en pro del desarrollo de la región y de esta manera dar a conocer nuestra gran diversidad de fauna y flora que se encuentra en el mirador los Currucuyes, mediante el uso de herramientas de marketing como son las redes sociales específicamente Facebook para acceder y promover el ecoturismo, de esta manera dar a conocer sus experiencias, comentar y poder generar otra fuente de ingreso para la comunidad en los municipios de Saboya y Chiquinquirá de departamento de Boyacá Colombia.

Al verse reducidos los recursos naturales, la sociedad debe adoptar la ciencia de la sostenibilidad, por lo cual el ecoturismo toma vital importancia como medida de protección de los recursos naturales, es por esto que este documento pretende mostrar cómo el diálogo de saberes a través de las redes sociales puede ser una herramienta para dar a conocer el estado en que se encuentran los destinos naturales de cada zona, fundamentado en el pensamiento de los diferentes grupos humanos y constituir una fuente de información diversa en el que se integre las fuerzas activas del mejoramiento y conservación del Mirador.

\section{PROBLEMÁTICA}

El sendero ecoturístico los Curucúyes, durante el recorrido se observo una gran diversidad de bosques con presencia de vegetación nativa perteneciente a los páramos andinos, destacándose algunos árboles como los robles grandes y frondosos que albergar gran variedad de aves, diversidad de pinos que por su aroma atraen insectos y mariposas de colores, arbustos como el tuno, campanillo y laurel que contribuyen a la producción y reserva del agua y son el habitad de muchos animales que habitan en las copas y se alimentan de sus semillas, también, se encuentran los legendarios frailejones con edades que sobrepasan los 300 años, por otra parte, un espectacular mirador desde el cual se divisa el occidente 
Boyacense tierra de leyendas y mitos, los cerros fura y tena que entre sus montañas esconde la muy apreciada esmeralda.

El recorrido comienza como punto de partida el sector la raya ubicado sobre la vía principal que comunica a Saboya con Barbosa Santander, se accede caminando por un tiempo aproximado de dos horas y cuarenta minutos a un paso normal por una carretera destapada, junto a la orillas del camino encuentra diversidad de frutos silvestres como moras, uvas montunas, anís de bosque, algunos lulos de selva caracterizado por sus vivos colores, con un sabor y textura inigualable como se visualiza en la Figura 1. Frutos silvestres Mirador Currucuyes, gran combinación de olores que despiertan los sentidos.

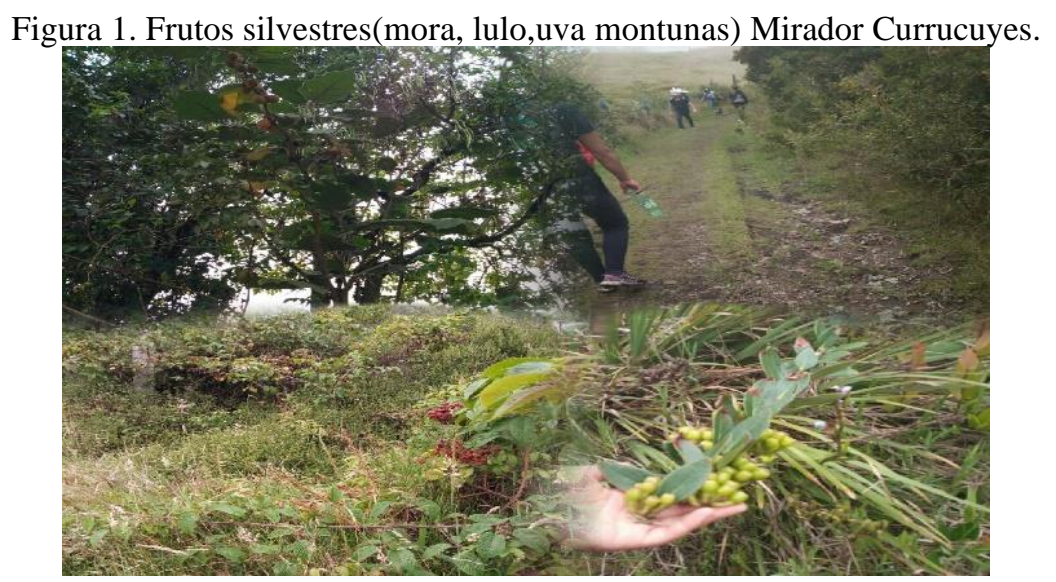

En el plan de desarrollo del municipio de Chiquinquirá, "UNIDOS POR CHIQUINQUIRÁ” 2012 - 2015,el sector turismo se tiene contemplado como una ruta turística al mirador de los Currucuyes, por el valor que representa la preservación de la vegetación, protección y conservación de las fuentes de agua existentes que provee a la población rural y las regiones aledañas, la Corporación Autónoma Regional(CAR) en convenio con el municipio de Chiquinquirá desarrollaron un proyecto de protección para preservar la vegetación y conservar las fuentes de agua allí existentes, debido a la aparición de mataderos clandestinos y el uso indebido de los suelos en el área rural que pone en riesgo la biodiversidad del sector, por el desconocimiento de las normas para la realización de la actividad turística de manera sostenible económica, cultural, social y ambiental, que cumpla con las con la debida señalización turística establecida por el Ministerio de Turismo que tenga demarcado durante su recorrido por medio de avisos, pancartas, carteles, imágenes, flechas, de igual forma, contar alertas de zonas de peligro que eviten riesgos a los visitantes en la cima del sendero se tengan barandas para que eviten el paso de los turistas a estas zonas de alto riesgo, así mismo, un punto de control el cual restringa la entrada a grupos de más de diez personas, el paso de mascotas al área protegida, que no permita el ascenso al páramo cuando este con neblina y se preste el servicio de guíanza turistas en época de lluvia por su gran altura y se den 
indicaciones de seguridad a la vez se cuente con información oficial ya sea documental en la que se pueda acceder a través de las redes sociales y en la página de las alcaldías.

El ecoturismo se está viendo como una medida de preservación y desarrollo para la región por razones como: la inclusión social ya que no proporciona oportunidades de empleo a la comunidad que vayan enfocadas a la reducción de la pobreza; con la aparición del internet como lo afirma Según Castells, M. (2001): “internet es más que una tecnología; un medio para todo medio de comunicación, de interacción, de organización social. Un medio en el que se basa una nueva sociedad en la que ya vivimos: la Sociedad en Red “, con la llegada de las redes sociales, blogs, portales de turismo, los consumidores del producto turístico podían exponer sus opiniones, afectando las decisiones de otros consumidores con otras partes del mundo sin salir de casa al compartir sus experiencias en la red a través de fotos videos, donde integra los conocimientos empíricos con los teóricos, hacen un intercambio de conocimientos para entender la realidad y producir diferentes interpretaciones (Acebedo, 2014).

En la actualidad las Tecnologías de la Información y las Comunicaciones(TIC) se ha convertido en un aspecto clave del siglo XXI en el sector turismo, siendo en muchos casos un factor relevante en la aparición de nuevos mecanismos o tendencias que hacen frente a las tradicionales, en donde las redes sociales específicamente la red Facebook ha servido para diferentes propósitos en el turismo en el que acceden a información, facilitan la búsqueda, comparten experiencias se comunican con clientes e intercambiar información entre individuos sin limitaciones de espacio y tiempo lo cual facilita la buscar la información independientemente del lugar donde se encuentre sobre destinos, precios, itinerarios y sitios de interés. Además de esto, hicieron reservaciones vía internet. Según Ericka Ortega (2010, permite al gran público contar con soportes de altas prestaciones que posibilitan el uso de servicios de geolocalización, acceder a potentes bases de datos e incluso implementar sistemas de realidad aumentada (Timothy, 2011). empleando el dialogo de saberes constituye un aprendizaje mutuo, el cual busca promover la construcción social del conocimiento mediante el intercambio de ideas, sentires, imágenes, creencias, nociones, conceptos, practicas, historias, deseos, vivencias y emociones para alcanzar la comprensión común y plenitud de la vida, se fundamenta en el respeto y el intercambio de palabras entre dos personas o más, al integran los conocimientos empíricos con los teóricos para entender la realidad y producir diferentes interpretaciones(Acevedo, 2014). Jaime Garzón político Colombiano decía "Para garantizar la realización de un dialogo de saberes, los procesos educativos deben constituir la más amplia participación de todos y todas sus integrantes no solo en la ejecución del diseño y conducción del proceso. Participar es incidir en las decisiones y no solamente ser activo en la ejecución de lo que otros deciden". 


\section{POLÍticas NACIONALES DE DESARROLlo SOSTENIBLE PARA LA ACTIVIDAD TURÍSTICA}

\subsection{POLÍTICA DE TURISMO DE NATURALEZA}

El sendero ecoturístico los Currucuyes por ser un área natural se encuentra tal como se menciona en la política de turismo para la naturaleza realizada por el Ministerio de Comercio, Industria y Turismo, la cual señala que se debe hacer una planeación adecuada para la conservación de la biodiversidad, para establecer el ecoturismo como estrategia de conservación, contribuyendo a cumplir con la función ambiental y social de los parques nacionales naturales, promoviendo la valoración social de la naturaleza y el reconocimiento del ecoturismo en los actores locales, regionales y nacionales como una alternativa que aporta a la conservación del patrimonio natural y cultural. en la figura 2 vayas de información acerca del Sendero de los Currucuyes, en los cuales se encuentra delimitada la zona de reserva.

\subsection{POLÍTICA PARA EL DESARROLLO DEL ECOTURISMO}

El sendero los Currucuyes es de carácter ecoturístico, allí se práctica las actividades de senderismo, avistamiento de aves además el ciclo montañismo, observación de fauna y flora, las cuales deben ser practicadas cuidadosamente para así mitigar los impactos negativos que deja el turismo, para minimizar estos impactos negativos se debe monitorear la vegetación, mediante inspecciones visuales y registros fotográficos, en los senderos se debe verificar el ancho de estos con ayuda de indicadores visuales que permitan ver el registro periódicamente es por ellos que se adecuo un adoquinado en ladrillo con alcantarillado, como también deben contar con torres de observación que se adapten a las condiciones del ecosistema sin alterar o perturbar la fauna que allí habita todo esto bajo un desarrollo sostenible que tenga en cuenta la comunidad insitu e integrando a los distintos actores tanto gubernamentales como privados.

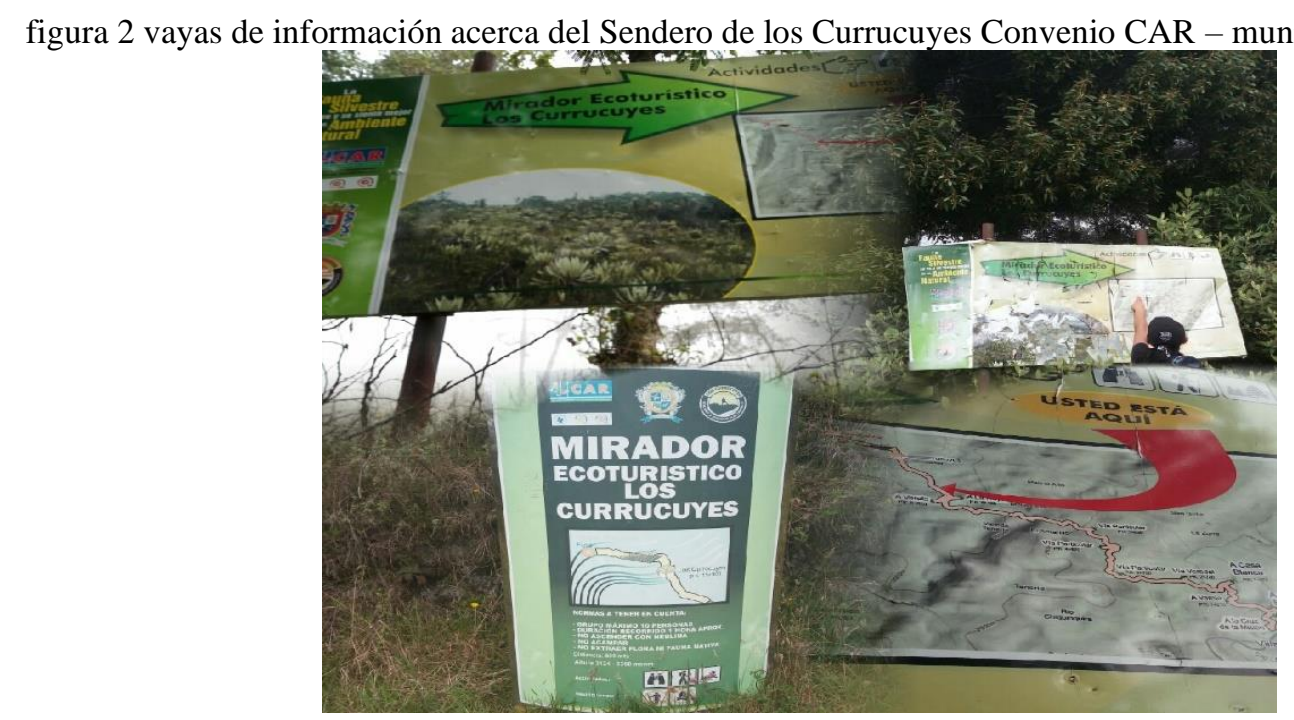


Se determina las responsabilidades de los actores locales de la región de acuerdo a la política para el desarrollo del ecoturismo (Ministerio de Comercio Industria y Turismo , 2012): Actores para la protección de estas áreas con distintas actividades y responsabilidades:

Custodio: Es la entidad o persona, de carácter público o privado, responsable de la protección y del manejo de las áreas naturales en las que se desarrollan actividades ecoturísticas, diseña mecanismos, controles y procedimientos para asegurar que se cumplan las normas tendientes al uso adecuado de dichas áreas. Operador: Es una compañía especializada en ecoturismo cuyo objetivo es diseñar productos ecoturísticos, debidamente inscrita en el Registro Nacional de Turismo. Promotor: promueve los productos en los mercados nacionales e internacionales y de apoyar a los operadores en el enlace con los mayoristas internacionales especializados en el tema. Prestadores de servicios: garantizan la calidad de estos, tienden a certificar los procesos de prestación de los servicios de modo que se acerquen a los estándares internacionales. Comunidad local: se incorpora principios y conceptos de la biología de la conservación y teniendo en cuenta el respeto por las identidades culturales, para que sean exitosos y viables a largo plazo. Ecoturista: Debe ser receptivo frente a las recomendaciones sobre las normas que se deben cumplir en el lugar y sobre la conducta que debe observar, poner en práctica los lineamientos establecidos en esta política, especialmente en lo que tiene que ver con evitar la depredación de los recursos y la contaminación del entorno. Otras instancias: la Unidad Administrativa Especial de Parques Nacionales Naturales, propondrá los criterios y las condiciones de negociación para facilitar la participación privada en la prestación de servicios ecoturísticos.

\section{METODOLOGÍA}

Se privilegio la investigación con enfoque cualitativo, por cuanto se orienta al diálogo de saberes con redes sociales, busca entender las causas del deterioro del atractivo, es decir "hacer una aproximación global de las situaciones sociales para explorarlas, describirlas y comprenderlas. Es decir, a partir de los conocimientos que tienen las diferentes personas involucradas en ellas y no deductivamente" (Bonilla, 1989, p.25). Con base en los conocimientos de las personas involucradas, el tipo de investigación que se desarrollara es la investigación acción participación (IAP), la cual es definida por Orlando Fals Borda, es un proceso abierto de vida y de trabajo, una vivencia progresiva de evolución hacia la transformación estructural de la sociedad y de la cultura como objetivos sucesivos y parcialmente coincidentes. Es un proceso que requiere un compromiso, una postura ética y persistencia en todos los niveles, fue pionera en proponer un diálogo de saberes, otro aporte fue pluralizar la reconstrucción del pasado introduciéndole más voces, con lo que se alteraba la narrativa tradicional occidental en la que hablaba solo el saber experto. (Archila M. 2017). Las fases de la IAP según Fals Borda son: 
Observación: Se realizó reconocimiento del lugar, al elaborarse un listado de factores como fauna, flora, clima y suelo entre otros, como también se identifican el estado en que se encuentra el mirador y el grado de cumplimiento de la políticas de desarrollo ecoturismo, un análisis histórico del atractivo a través de la observación directa y de entablar dialogo con los habitantes y turistas que visitan el lugar para conocer su opiniones, así mismo, se seleccionó un grupo de personas mediante un focus group se recoge información relevante mediante el dialogo saberes para conocer aspectos importantes y las características intrínsecas de la zona.

Diagnóstico: Con la información recolectada acerca del lugar las entidades gubernamentales, territoriales y los habitantes de la región a fin de conocer el estado y las respuestas dadas por las personas entrevistadas junto con las evidencias y la información del lugar registrado en documentos,videos, archivo fotográfico, audios en el que permite conocer las situaciones insitu de los problemas que tiene la comunidad

Planificación: Luego se realiza un plan de acción en el que se diseñan una serie de acciones a fin de solucionar los problemas dando cumplimiento con lo estipulado con las normas ecoturísticas y la políticas de desarrollo ecoturístico en el que se hizo uso de redes sociales Facebook y se establece interacción entre la comunidad, las fuerzas activas y la Universidad.

Acción: Actuación para poner en práctica el plan implementando las redes sociales se creó una página en Facebook en marzo de 2019 con el nombre del atractivo "Mirador Ecoturístico Los Currucuyes", esta página en un mes alcanzo veinte visitas, veintidós me gusta a la página, dos recomendaciones, veintidós seguidores, veintiuna interacciones con las publicaciones, cuarenta y cuatro reproducciones de los videos. Se hicieron publicaciones de videos con fotos de la flora que crece en el área el cual ha tenido una alcance de ciento ochenta y tres personas, nueve interacciones y ha sido compartida tres veces, así mismo se publicó un álbum de fotos del mirador el cual tuvo una interacción de seis personas un alcance de nueve

Evaluación: mediante la red social Facebook se realizó promoción acerca del lugar en el que se evidencio la importancia que tiene el sitio turístico para la región como también la importancia de promover el turismo de una forma responsable.

Experiencia de promoción en redes sociales (Facebook) Por medio de la red social Facebook se hizo publicación acerca del lugar por medio de fotos, videos, álbum de fotos, lo que permitió que muchos habitantes de la región y sus alrededores despertar la curiosidad para que visitaran el lugar y en otros casos los usuarios en sus comentarios preguntaron como hacían para llegar al lugar, para lo cual se les fue dando respuesta a las inquietudes, se fue documentando, proporcionando información del lugar con indicaciones básicas, restricciones que debe tener cuando vayan a ingresar al mirador. 
Con relación al análisis de la informacion publicada en la red social Facebook se evidencio que la actividad que más visitas fue el video con 193 y luego la foto que contenía el mapa con la ubicación con un alcance de 166 visitas de las cuales los usuarios reaccionaron y comentaron otra actividad las dos actividades como se muestra en la figura 3 .

$\mathrm{Al}$ implementar el dialogo de saberes con la comunidad, los turistas que han visitado el lugar opinaron que les pareció muy bonito, y los representantes de la comunidad vieron una oportunidad económica en el turismo, a partir de las publicaciones realizadas en las redes sociales de las personas que han ingresado sugieren al gobierno de estos dos municipios que deben implementar las políticas de desarrollo ecoturismo para que el mirador sea un atractivo sostenible y sustentable, para que de esta manera sea potencializado el mirador a nivel local, regional, nacional e internacional.

la figura 3. Actividades de la interacción con la red social Facebook.

\begin{tabular}{|c|c|c|c|c|c|c|c|c|c|c|}
\hline \multicolumn{5}{|c|}{ Pagina de facebook } & \multicolumn{6}{|c|}{ Pagina de facebook } \\
\hline $\begin{array}{l}\text { Fecha de } \\
\text { publicacion }\end{array}$ & Publicacion & Tipo & Alcance & Interaccion & $\begin{array}{c}\text { Fecha de } \\
\text { publicacion }\end{array}$ & & Publicacion & Tipo & Alcance & Interaccion \\
\hline \multirow{2}{*}{ 4/5/2019 } & \multirow{2}{*}{$\begin{array}{l}73 \text { jicacion con foto de mapa: } \\
\text { herramienta google maps }\end{array}$} & \multirow{2}{*}{ foto } & \multirow{2}{*}{166} & 16 & $4 / 5 / 2019$ & \multicolumn{2}{|c|}{$\begin{array}{l}\text { 3icacion con foto de mapa: } \\
\text { herramienta google maps }\end{array}$} & foto & 166 & 3 \\
\hline & & & & 13 & \multirow[b]{2}{*}{$4 / 3 / 2019$} & \multirow[b]{2}{*}{$\infty$} & \multirow[b]{2}{*}{ Estado } & \multirow[b]{2}{*}{ Estado } & \multirow[b]{2}{*}{17} & $\frac{6}{3}$ \\
\hline $4 / 3 / 2019$ & Estado & Estado & 17 & $\frac{4}{3}$ & & & & & & 0 \\
\hline \multirow{2}{*}{$3 / 30 / 2019$} & \multirow{2}{*}{ video } & \multirow{2}{*}{ video } & \multirow{2}{*}{193} & 4 & \multirow{2}{*}{$3 / 30 / 2019$} & \multirow{2}{*}{ 酯 } & \multirow{2}{*}{ video } & \multirow{2}{*}{ video } & \multirow{2}{*}{193} & $\frac{5}{0}$ \\
\hline & & & & 8 & & & & & & 3 \\
\hline \multirow{2}{*}{$3 / 19 / 2019$} & albun de fotos & \multirow{2}{*}{ foto } & \multirow[t]{2}{*}{0} & 0 & \multirow{2}{*}{$3 / 19 / 2019$} & $\approx$ & & foto & 0 & 5 \\
\hline & & & & $\begin{array}{c}5 \\
17\end{array}$ & & $\infty$ & albun de fotos & foto & 0 & $\frac{0}{0}$ \\
\hline $3 / 11 / 2019$ & albun de fotos & foto & 9 & 2 & 31112019 & wh & Albu de foto & foto & 9 & 2 \\
\hline $3 / 11 / 2019$ & albun de fotos & foto & 0 & 0 & $3 / 11 / 2019$ & $=$ & dibun de totos & Toto & 9 & 0 \\
\hline & & & & 4 & $3 / 11 / 2019$ & 5 & albun de fotos & foto & 0 & $\frac{4}{0}$ \\
\hline & Clics en publicaciones & & & & & & & & & 0 \\
\hline & Reaciones, Comentarios y veces & que se & & & & & $\begin{array}{l}\text { Reacciones } \\
\text { Comentarios }\end{array}$ & & & \\
\hline & ha compartido & & & & & & Veces compartido & & & \\
\hline & Alcance & & & & & & Alcance & & & \\
\hline
\end{tabular}

Las redes sociales han tenido gran importancia en la promocion y divulgacion del ecoturismo debido a la gran diversidad de contenidos interactivos que se pueden realizar, ya sea, de voz, datos, videos acerca del mirador turistico los Currucuyes informacion extraida de la comunidad local al aplicar el dialogo de saberes se conto con diferentes personas de la comunidad a fin de recoger la realidad y contribuir con soluciones que promuevan el desarrollo de la region de una manera sostenible junto con los aportes y recomendaciones que hicieron los turistas a traves de los comentarios en las redes social a partir de sus vivencias. 


\section{REFERENCIAS}

Biocultural CONE. Diálogo de saberes para el replanteamiento teórico de la restauración ecológica con enfoque biocultural. 2013.

Blanco PC, Forest P. Diagnóstico de los potenciales turísticos para el diseño de senderos eco turísticos. Cao Bosque. 2017;13:28-43.

Caro JL, Luque A, Zayas B. Nuevas tecnologías para la interpretación y promoción de los recursos turísticos culturales. 2015;13:931-945.

Luna EP. Diálogo De Saberes Y Proyectos Dialog Of Knowledge And Research Projects In School. 2008.

M GL, Laura U, Marcovecchio MJ, Margarit V. Espacios Dialógicos con Tecnologías de la Información y Comunicación ( TIC ) como Portadores de Conocimientos en Construcción en el Aprendizaje en Carreras Universitarias. 2015:1209-1212.

Marín LF, Siles I, Daza S, Arboleda T, Rodríguez JA, Torres CR. Diálogo de saberes. :33-45.

Ortiz A, Cynthia HW. Redalyc. Tecnología de la Información y la Comunicación el Turismo. Análisis de la producción académica en revistas brasileñas. 2016.

Profile SEE, Profile SEE. Ecología y bioeconomía. El diálogo de saberes 2017; November doi: 10.21676/23897848.2086 2017

Sustentable EP, Autónoma U, Jiménez ES, Morfín CM, Morfín CM. Diálogo de saberes Knowledgeable dialogues through the tourist activity. 2011.

Turismo DEL. Asistencia técnica en planificación Del turismo.

Vila D, Vila A. Redalyc.gestión de las redes sociales turísticas en la web 2.0. 2014.

Vivas ca. Diseño e implementación de senderos ecoturísticos como opción de desarrollo local : caso sendero ecoturístico la zarza municipio de Duitama - Boyacá.

Bernal Poveda Campo, tesis doctoral "turismo de naturaleza y cultura en el departamento de Boyacá (Colombia). marco normativo y estratégico para la innovación turística en el territorio", Universidad de Salamanca, España 2015

Máster en Periodismo de viajes. Director de desarrollo de negocios en 1492, travel. 2013; xiv: 33-45. Diálogo de saberes a través de la actividad turística 1. 2011.

Cómo entender el diálogo de saberes. 2017:61-62. 